\title{
Understanding older adults' use of social technology and the factors influencing use
}

\author{
Gemma Wilson $^{1 \star}$ (D), Jessica R. Gates ${ }^{1}$ (D), Santosh Vijaykumar ${ }^{1}$ (D) and Deborah J. Morgan² (D) \\ ${ }^{1}$ Faculty of Health and Life Sciences, Northumbria University, Newcastle upon Tyne, UK and ${ }^{2}$ Centre for \\ Innovative Ageing, College of Human and Health Sciences, Swansea University, Swansea, UK \\ ${ }^{\star}$ Corresponding author. Email: Gemma.wilson@northumbria.ac.uk
}

(Accepted 23 March 2021; first published online 20 April 2021)

\begin{abstract}
Having access and skills to use social technology, i.e. social internet use, social media and social applications, are considered as being vital to online social connection. Whilst evidence exists around facilitators and barriers to general technology use, evidence is limited with regards to the motivators, skills and tangible offline benefits older technology users experience with social technology. Therefore, this study used a qualitative, exploratory method to understand older adults' experiences of using social technology to connect with others. Semi-structured interviews were conducted with 20 older adults (65+ years) across England, Scotland and Wales. Despite having access to social technology for social connection, and using this technology regularly, multiple barriers impacted motivators and skills for use, namely perceived self-efficacy and fear, the culture of online communication, absence of social capital and physical functioning. Some of these barriers of social technology use are reminiscent of barriers of wider technology use and emphasise the importance of addressing these barriers for digital exclusion, as well as social connection. However, some of these barriers were specific to social technology use and should be considered when providing guidance or interventions to increase older adults' online social connection. Social connection was a clear tangible outcome to social technology use, and individuals discussed the benefits of using social technology, particularly visual communication tools, for online connection.
\end{abstract}

Keywords: ageing; digital; technology; social media; loneliness; social isolation; digital exclusion; digital divide; gerontechnology

\section{Introduction}

With rapid technological change, the advent of smart mobile technology has resulted in digital devices, social media and the internet becoming commonplace for many. This technology has become ubiquitously used as a means of social connection and has been suggested as a potential solution to both alleviate and prevent loneliness in later life (Ballantyne et al., 2010; Leist, 2013; Holttum, 2016).

Evidence suggests that social connection is one of the key reasons for technology use, specifically as a means to keep up with friends and family (Winstead et al., 
2013; Tsai et al., 2015; Ferreira et al., 2016). For some, motivations to achieve social connection through the use of 'social technology' (i.e. the online resources that allow individuals to connect with others; Nowland et al., 2018) can be related to feeling a sense of wider social inclusion (Winstead et al., 2013; Ferreira et al., 2016; Coelho et al., 2017; Tsai et al., 2019). Although, this evidence has been contradictory, and for some, online social activity does not always equal 'digital togetherness' (Marino, 2015). Contradictory evidence also exists with regards to the effectiveness of technology on alleviating loneliness (Beneito-Montagut et al., 2018). Several studies, including Cotten et al. (2013), have identified a positive effect between technology use and a reduction in loneliness. Similarly, Ballantyne et al. (2010) reported decreased feelings of loneliness associated with the use of social media sites, while Tsai et al. (2010) found that short weekly video conferencing with family members decreased loneliness over a three-month period. In contrast, other studies found no relationship between technology use and levels of reported loneliness (White et al., 1999, 2002; Slegers et al., 2008; Burholt et al., 2020).

These contradictory findings may, in part, be explained by findings from Nowland et al. (2018) who present a theoretical model which illustrates this relationship as being both bidirectional and dynamic, identifying variants of outcomes to technology use and loneliness. Using both the displacement hypothesis (Kraut et al., 1998; Nie, 2001; Nie et al., 2002; Valkenburg and Peter, 2007) and the stimulation hypothesis (Gross, 2004; Valkenburg and Peter, 2007), the authors identified that when individuals use technology as a means of displacing offline social relationships with online social relationships and escaping the social world (i.e. the displacement hypothesis), loneliness increases, however, when the internet is used to support or enhance existing or new social connections (i.e. the stimulation hypothesis), loneliness is reduced (Nowland et al., 2018). Nowland et al. (2018), along with other studies, have identified that older people are more likely to use technology to maintain their existing relationships with family and friends (Xie, 2007; Cotten et al., 2013; Nowland et al., 2018). Yet many studies have not considered social support as a mediator of successful utilisation of technology to reduce loneliness, unlike Heo et al. (2015), who reported that higher levels of internet use were associated with higher levels of social support and lower levels of loneliness, although the direction of this relationship is unclear.

Over the last decade, there has been a notable rise in internet use, with more than twice as many adults in the United Kingdom (UK) over 75 years old using the internet in 2018 than in 2011 (Eurostat, 2017; Office for National Statistics, 2018). However, older adults are still considered as being 'digitally excluded' and use the internet to a lesser extent than younger generations (Age UK, 2018). For example, in the UK, only 27 per cent of adults over 65 years old use social media, compared to 96 per cent of 16-24 year olds (Office for National Statistics, 2018). Similarly, only 37 per cent of adults over 65 years old in the United States of America use social media compared to 88 per cent of 18-29 year olds (Smith and Anderson, 2018). Evidence shows that older adults are still more likely to use the internet more 'narrowly' than younger generations, with older adults predominantly using the internet for information finding or sending emails (Office for National Statistics, 2018). 
Digital exclusion is defined as the absence of digital skills, connectivity and accessibility (Government Digital Service, 2014), meaning that even for those with access to a computer and access to the internet, digital exclusion is not as straightforward as those who do use technology and those who do not use technology (Büchi et al., 2016; Age UK, 2018). In fact, digital exclusion is now recognised as existing at three levels: access, skills and usage, and the tangible outcomes from internet use which can result in offline benefits, specifically economic, social, political, institutional and educational benefits (Blank and Groselj, 2014; Van Deursen and Helsper, 2015; Scheerder et al., 2017). With the focus of this study being social connection, facilitating social connection constitutes one of the tangible offline benefits of technology in this instance (Van Deursen and Helsper, 2015). However, despite the evidence promoting technology use as having tangible offline social benefits, to date, all evidence of digital exclusion refers to barriers of using technology generally, as opposed to focusing on exclusion and barriers to social technology specifically.

General digital exclusion results in individuals having inequitable capacity to participate fully in society (Schejter and Tirosh, 2015) and can heighten existing inequalities in varying ways, e.g. those not logging on to online services lose out in applying for eligible parking concessions, accessing benefits and access to housing (Age UK, 2018). This inequality is twofold, as whilst digitally excluded older adults are likely to experience heighted inequalities as a result of being digitally excluded, those suffering inequalities are most likely to experience digital exclusion in the first instance; specifically, lower-economic status (Gracia and Herrero, 2009; Cresci and Jarosz, 2010; Choi and Dinitto, 2013; Age UK, 2015; Ihm and Hsieh, 2015; Yu et al., 2016; Hargittai and Dobransky, 2017; Hargittai et al., 2019; Matthews et al., 2019), lower educational attainment (Cresci and Jarosz, 2010; Neves et al., 2013; Yu et al., 2016; Bergström, 2017; Hargittai and Dobransky, 2017), increased age (Gilleard and Higgs, 2008; Choi and Dinitto, 2013; Friemel, 2016; Yu et al., 2016; Bergström, 2017; Hargittai and Dobransky, 2017; Gordon and Hornbrook, 2018; Matthews et al., 2019; Yoon et al., 2020), health status and disability (Cresci and Jarosz, 2010; Choi and Dinitto, 2013; Age UK, 2015, 2018; Yu et al., 2016; Matthews et al., 2019) and gender, with women generally being more digitally excluded than men (Age UK, 2015, 2018; Matthews et al., 2019), although recent evidence does also contradict this (Yu et al., 2016).

As well as societal inequalities that influence digital inclusion, there are specific barriers that also have an impact on general technology use. Two of the most influential factors negatively impacting online activity are self-efficacy and fear (Age UK, 2015, 2018; Tsai et al., 2015; Centre for Ageing Better, 2018). Particularly for older adults, self-stereotyping may become a self-fulfilling prophecy which can have an impact on internet use (Pennington et al., 2016). Physical factors, such as dexterity or eyesight problems, can also impact overall technology use, especially when using smaller tablets or mobile phones (Neves et al., 2013; Olphert and Damodaran, 2013). Research has found significantly lower rates of computer and internet usage among older adults with disabilities (Wright and Hill, 2009; Gell et al., 2015). Those with impaired eyesight, hearing, mobility and hand/finger dexterity, and those that are housebound, had usage rates of 17 per cent, compared to those without disabilities who reported usage rates of 37 per cent (Wright and Hill, 2009). The study by Gell et al. (2015) was one of the few studies looking at 
the use of technology for communication (namely sending emails and text messages) among internet use for other reasons. The authors found that older adults with more severe physical disabilities, which required them to access help for performing activities of daily living or mobility outside the home, used technology for communication at a significantly lower rate. However, the authors also found those experiencing persistent pain or breathing difficulties used technology frequently, perhaps due the opportunity to access health information or for social connection (Gell et al., 2015).

Moreover, existence of social capital perpetuates digital inclusion, and supports evidence which highlights the importance of social ties for older adults (Berkman et al., 2000; Cornejo et al., 2013). Those who have existing social support were more likely to be using technology due to supported initiation and supported maintenance or troubleshooting (Tsai et al., 2015; Van Deursen and Helsper, 2015; Friemel, 2016). As well as initial interest and initiation, there is influence on older adults' adoption of these technologies through critical observational learning from friends and family members (Tsai et al., 2015).

Whilst the barriers of general technology use are now well recognised for older adults and other age groups, the vast majority of this evidence refers only to general technology, e.g. evidence reviews focusing on digital exclusion include barriers to accessing travel services, civic participation, reading online newspapers or books, and finding information about goods or services (Age UK, 2018). This current evidence base does not specifically ascertain the facilitators and barriers of social technology use which support online social connection. Therefore, this study used a qualitative, exploratory method to explore older adults' experiences of using social technology (including digital devices, social media and social applications) to connect with others. This study involved older adults with existing digital access (firstlevel digital divide), and specifically aimed to understand:

- Older adults' skills (second-level digital divide) in using digital devices, social media and social applications as tools to connect with others.

- The motivators and tangible outcomes (third-level digital divide) of using digital devices, social media and social applications as tools to connect with others.

\section{Method}

\section{Design}

This paper presents one phase of a larger study in which a mixed-methods two-phase exploratory sequential design (Creswell et al., 2003) was adopted. In line with this design, qualitative data were initially collected and analysed, informing a quantitative second phase. This study presents the findings from this first, qualitative phase. This study received ethical approval from Northumbria University's ethical approval system.

\section{Participants}

In order to participate, participants must have been: (a) aged 65 or above and (b) use, or have used, digital devices, social media and/or social applications (e.g. text 
Table 1. Participant demographics

\begin{tabular}{lcc}
\hline Demographic information & N & $\%$ \\
\hline Age (years): & 12 & 60 \\
\hline $65-74$ & 8 & 40 \\
\hline $75-84$ & & 60 \\
\hline Gender: & 12 & 40 \\
\hline Female & 8 & \\
\hline Male & & 55 \\
\hline Country: & 11 & 10 \\
\hline England & 2 & 35 \\
\hline Scotland & 7 & \\
\hline Wales & & 40 \\
\hline Marital status: & 8 & 30 \\
\hline Bereaved & 6 & 5 \\
\hline Married, civil partnership, co-habiting & 1 & 25 \\
\hline Separated or divorced & 5 & \\
\hline Single & & \\
\hline
\end{tabular}

messaging services, email or video calling applications) to connect with others. Twenty participants were recruited across England, Scotland and Wales (Table 1).

\section{Procedure and data collection}

To achieve wide-reaching representation, a number of recruitment strategies were used. Initially, the study was advertised on various social media platforms. Furthermore, relevant third-sector organisations and public libraries across the UK were approached to assist with advertising the study. All advertising and project information was available in English and Welsh languages. A snowball sampling strategy was also used and proved particularly effective, with several participants being referred to the research team by existing participants.

If interested, potential participants approached the research team either by email or telephone, and they were then provided with a detailed participant information sheet and consent form (available in both English and Welsh languages). Potential participants also had the opportunity to ask any questions. If the participant wished to proceed, a date and time were arranged to conduct the interview, either by telephone, face-to-face or using Skype, depending on the participant's preference.

Semi-structured interviews were conducted with participants using the semistructured interview schedule (Figure 1). The interviews explored the individual's own perspectives, developing insight into the motivations, facilitators, barriers and experiences of using technology. Interviews were audio recorded using a Dictaphone and were transcribed verbatim. 
General introductions, introduce the purpose of the interview and answer any additional questions before proceeding.

1. Please tell me about your knowledge of/use of technology generally.

2. Please tell me if/how you use technology to communicate with others. Do you use social media?

Do you use visual communication tools such as Skype/FaceTime? Other?

3. Please tell me about other ways (if any) that you connect with others. E.g. do you attend social groups? Do you meet people face-to-face or over the telephone?

4. Who instigated your use of technology as a form of communication? Did you try it yourself, did your family/friends suggest this, or something else?

5. How long have you been using technology to communicate with others?

6. How do you feel about using technology to communicate with others? Are there any facilitators or barriers to its use? Is it easy/difficult? Is this the same, better or worse than meeting someone face-to-face?

7. Has the use of technology changed the way that you communicate with others? If so, how?

Thank participants and end interview.

Figure 1. Interview schedule.

\section{Data analysis}

All interview transcripts were analysed using thematic analysis, adhering to the six steps set out by Braun and Clarke (2006): familiarising yourself with the data; generating initial codes; searching for themes; reviewing themes; defining and naming themes; and producing the report. The NVivo 12 software package was used to facilitate analysis of this data.

\section{Findings}

Two themes were generated from the qualitative data, each with their own subthemes (Figure 2): the facilitators and barriers of social technology use; and technology as a tool for social engagement. 


\begin{tabular}{|l|l|}
\hline Facilitators and barriers of & Perceived self-efficacy \\
\cline { 2 - 2 } social technology use & Fear \\
\cline { 2 - 2 } & Culture and communication \\
\cline { 2 - 2 } & Social capital \\
\cline { 2 - 2 } & Physical functioning \\
\hline \multirow{2}{*}{$\begin{array}{l}\text { Technology as a tool for } \\
\text { social engagement }\end{array}$} & Technology as a connector \\
\cline { 2 - 2 } & The importance of the visual \\
\hline
\end{tabular}

Figure 2. Themes and sub-themes.

\section{Facilitators and barriers of social technology use}

\section{Perceived self-efficacy}

Despite all participants being users of digital devices and social media, many perceived themselves as being novices, and not being 'technology minded' (P005, female, 79 years). Particularly, P002 had low confidence, low perceived self-efficacy, and a lack of patience with her devices, social media sites and applications. This directly influenced her use of social technology, and directly influenced online social connection:

To be honest I just didn't have patience with [Facebook]. (P002, female, 78 years)

I think really, it's the fact that I am starting feel left out because I don't know it and I can't cope with it and I just ... It annoys me that I can't take it in. I mean why not? I mean there is no reason for me not to be able to. As I say I don't think I'm thick at all, no it's just ... lack of patience, that's what I put it down to anyway. (P002, female, 78 years)

One participant described how rapid technological progression made him feel left behind:

Technology now flies over my head, I used to keep up with it but now ... it has to be said, technology has flown past me to a certain extent. (P011, female, 74 years)

Higher levels of self-efficacy were often related to early adoption. Many of the participants described their own use of computers in the workplace, and they believed their own familiarity had a positive impact on their current use:

I wouldn't say that I regard myself as a geeky type of person but my first computer, I bought probably 40 years ago now almost. It was very much in its infancy and I actually built the computer to start with. (P020, male, 65 years)

This was reflected in lower levels of self-efficacy by individuals who did not consider themselves as early adopters and had begun using a digital device later in their life: 
I'm liable, the occasions that I do go on [a desktop computer], I have to scream, help, why can't I get this? Why can't I get that? I don't like [the desktop computer]. I've not grown up with one. I have persevered with [the iPad] and I can adapt. I don't need any [thing other than the iPad]. (P018, female, 78 years)

This perception of low self-efficacy through later adoption had an impact on general technology use, which also influenced use of social technology.

Ageing was also considered to be a factor determining lower self-efficacy and lack of familiarity. Participants considered others of a similar age or older as having less understanding or less experience of using computers, mobile technology, the internet, and social media and applications than younger people:

I know a lot of friends of my age hardly use mobile technology or computers. They just feel very uncomfortable and don't know what they are doing. (P017, male, 76 years)

There was also some consideration of the participants' own future ageing and its potential to impact their own future use of technology:

I don't know if there will come a time as I get older when I drop out because I feel I can't keep up with it anymore. I don't think I will. I don't think that the pace is, you know, beyond me. (P019, male, 67 years)

Perceived self-efficacy had a direct impact on the use of technology for online social connection. Higher levels of self-efficacy were related to early adoption, with individuals who were more familiar with computers during their working years having higher levels of confidence than those picking up digital devices for the first time after retirement. Ageing was considered by the participants as being detrimental to technology use and reduced self-efficacy.

\section{Fear}

Some participants were fearful of using digital devices, social media sites and other applications, in case they broke them:

When I had the computer, I was really sort of nervous. I was thinking it would all crash or cease up or something would go terribly wrong with it, but that's not a fact is it? It's very rare that you sort of go badly wrong. You can usually sort things out. There is a little bit of fear I think of new technology particularly with the older age group. (P013, female, 79 years)

This fear prevented general technology use, and therefore also prevented the use of technology for social connection.

When using social media or generally being online, fear was also experienced through concerns of their own privacy and security. Some were concerned when posting information on social media sites and feared losing their privacy:

I don't want everyone to know what I am doing, when I am doing it kind of thing.

I think we're more cautious. (P002, female, 78 years) 
There was also fear surrounding security, and being hacked, when using social media sites, but also using the internet more generally:

One thing you do sort of wonder about the technology, how many people can hack in and get to know your business. I'm very wary about internet banking for instance. I don't quite trust that at the minute. (P013, female, 79 years)

Whereas this changed use of the internet and mobile applications for some, others used work-around strategies to confront these issues:

Talking about doing my banking online, I got a scam email and I thought this isn't right, so I printed it off and took it down to the bank, and because I had printed it off I was able to see things that you wouldn't see on the screen. They could see that it came from Russia. (P005, female, 79 years)

Fear had an influence on individuals' use of the internet, social media and other applications. This fear surrounded breaking the technology, privacy concerns and security issues.

\section{Culture and online communication}

There were often instances in which the perceptions of, and culture around, communication impacted the way individuals used social technology to communicate with others. Most participants felt that social technology, in all forms, was a useful tool to keep up to date with others, but that it did not replicate spending time with one another. Participants described digital social connection facilities as 'just a tool' (P003, male, 70 years) and as 'shortcut when you need it' (P006, female, 70 years):

More crucially is not allowing technology to be the be all and the end all. There is that need for people to always have people. (P020, male, 65 years)

Some individuals felt that online and telephone communication was not a way of conversing with one another but, instead, was a method of checking up on a person:

Having a person sitting in front of you and talking to you is much better than having a ten-minute phone call with somebody saying, you know, 'How are you? You're okay, are you?', you know just checking up like that. It's much better having a conversation, having somebody around for tea or having somebody in the house for an hour. (P011, female, 74 years)

One of the most salient issues discussed in terms of social technologies as replacing face-to-face communication was the perceived differences across generations, with individuals discussing the different views across their family:

My granddaughter is pregnant, the baby is due anytime now so there is sort of a running commentary going on all the time ... I find that good, but I do find sometimes you'll get a text message when a phone call would have been nicer. (P013, female, 79 years) 
Participants described their decision to communicate online was because they felt it was a less-intrusive form of communication when their families led busy lives and may not have time to talk to them:

You don't want to be intruding if you're ringing up you don't know, is it a good time or whatever. So, in those instances you can send an email or something on Messenger and then when they're ready, they can respond. So that's a good thing. (P016, female, 66 years)

There is always fear that you are interrupting people when you ring. (P019, male, 67 years)

Individuals overwhelmingly preferred face-to-face communication and perceived online communication as a supplementary tool to maintain relationships, as opposed to replicating other forms of communication, with one participant articulating social technology as having 'destroyed the essence of the human being' (P008, male, 77 years).

Individuals differed in the way that they communicated online, with some being more active and others more passive users of social media. There was also discussion of the moral attitudes to online communication, including fear of miscommunication online, that would not necessarily occur 'offline'. It was evident that despite all participants in this study using social media in some way, the way in which they used it differed considerably. Some individuals were more active than others in posting on social media:

Occasionally if I'm on holiday I might put a photo on, but that's about it. (P016, female, 66 years)

Even those actively posting on social media were cautious of what they posted and preferred more direct forms of online communication with family or friends for more 'private' matters:

I will message people if it is a private message then I would just message someone, it doesn't go public. (P003, male, 70 years)

For others, whilst they kept up to date with online profiles, they did not actively share their own information:

I mean I know that there is this thing with Twitter that I am not involved in. I don't tend to post. I read posts from other people. (P006, female, 70 years)

Whereas some individuals actively used social media themselves, others used it as a way of keeping up with others. Social media use was very personal, with individuals using the platforms in varying ways, however, both active and passive users reached the intended purpose, which for most participants was to keep up to date with the lives of their family and friends. 
Participants' active or passive style of social media use sometimes arose as a result of their attitudes to others' use of communicating online and use of social media:

People being very nasty. Sexist, racist, homophobic, you know, in the end I just cut ... I came off that group. Even though I'm missing out on some bits and pieces, it just wasn't worth it. (P006, female, 70 years)

There was also a fear of their own miscommunication when using online communication, compared to when communicating face-to-face:

If they send me a text or I send them a text, it can be very abrupt and misinformed. (P008, male, 77 years)

If you're not very careful the way you write an email can come across as a bit harsh ... In terms of the way you express yourself, which wouldn't come across the same way if you were communicating that verbally. (P016, female, 66 years)

Individuals' attitudes towards online communication, including communication from others, and worrying about misinterpretation, influenced their own use of online communication tools.

\section{Social capital}

Social capital refers to a social network with valuable benefits. Although definitions of social capital differ, one definition of social capital describes it as 'the features of social organisation such as networks, norms, and social trust that facilitate coordination and cooperation for mutual benefit' (Putnam, 1995: 67).

It was clear that existing social capital was highly influential in initiating use of digital devices, social media and other applications, and these networks enabled individuals to become familiar with this technology. The influence of social capital did not end at initial use of these digital tools, but it was also an important factor during ongoing support and maintenance of using digital devices and social media.

Many participants described their use of digital devices, social media and other applications as being initiated by others. For some, they had not used any digital device before being introduced to this through family members, and occasionally friends, who bought the devices for them, or supported them to get started:

Somebody bought me an [iPad] and then I felt obliged to use it. I don't think I would have bothered buying one for myself. (P002, female, 78 years)

I have a grandson who has just recently moved to London who knows more or less ... keeps me up to speed on things. (P009, male, 80 years)

Family members also introduced individuals to, or physically set up, social media platforms and text messaging applications, for the purpose of connecting with them and the rest of the family: 
My grandson put me on to Facebook because I've got one ... I've got a grandson in Australia and I just wanted to [connect with him] and my granddaughter has put us on a little group with WhatsApp. (P013, female, 79 years)

One individual also discussed the way in which she felt pressured into using social media by her daughter as it was the only way she could stay connected with them whilst on holiday. She felt this was the method her daughter used to 'force' her into using 'modern technology' (P004, female, 73 years):

I was very reluctant to go on Facebook initially and just for my children really especially my eldest daughter, it wasn't like a forced, you must get on Facebook, but it was ... She was on holiday in New Zealand and she was posting photographs on Facebook. She said, if you want to see them you are going to have to go on Facebook ... I suppose it was a twisted arm to get me on Facebook ... I think they force you in a way, don't they, to get into modern technology. (P004, female, 73 years)

Many individuals only began using digital devices, or social media, because of their existing social networks. The existence of social capital was of great importance for initiating use of digital tools for social connection.

The importance of an individual's social network did not end at technology initiation, but individuals also relied on this social capital to support them when learning something new, or to support them if there was a technical issue. Individuals often asked family members for help when they were not familiar with a digital device or social media platform, and they did not want to use it without support:

I can always pick up the phone and say, 'What's all this? How does this work?' I'm not afraid to ask the family, but I do tend to sort of ask them to show me how to do it rather than experiment. (P012, male, 71 years)

Some participants described using technical support from someone other than a family member. These individuals described seeking support from a high street shop, or using library services, when they had trouble with their digital devices:

I'm a bit uncertain about some of the security stuff. I think I've got my settings set as they need to be for privacy and all that. I did go to a session in the library. (P016, female, 66 years)

Not everyone relied on others and some individuals did feel that they had the skills to help themselves, and some were able to help others to improve their technical issues:

I used to sit in the library and people used to come to me and ... 'Help me. How do I do this and how do I do that?' (P011, female, 74 years)

It was not just immediate technical support that was considered. There was also concern when forecasting their own use of digital devices, the internet, social 
media and applications without continued support from family. This future loss of social capital was perceived as having an impact on future technology use, and therefore losing opportunities currently held:

I don't know if I would be nervous in doing some things because I've never had to do it, I suppose ... I tend to leave it if somebody else will do it for me and I do find that is a handicap because if anything happens to [my husband] I don't know how ... I would have to get the kids to do it. (P018, female, 78 years)

Individuals typically relied on their social networks to support them when learning something new, or when a technical issue needed dealing with. In a few cases, individuals described finding an external organisation to support them or doing this themselves. Once more, existing social capital was of great importance for continued use of technology.

\section{Physical functioning}

One further barrier to using digital devices was due to individuals' physical functioning, typically eyesight and persistent pain. Some participants chose larger devices, such as a tablet over a smartphone, due to the size of the screen:

Then I'm having a problem with my eyes at the moment, I've got to have cataracts done so I find the tablet better to see as well. (P001, female, 83 years)

Persistent pain, particularly in the fingers and wrists, was also a problem and impacted the way in which individuals used digital devices:

[The iPad] is bigger for my fingers. (P008, male, 77 years)

This had some sway on the device purchased, or the devices individuals could use. However, small buttons and small on-screen text had a direct impact on connecting with others online via social media sites and text messaging:

I just read other people's [comments on Facebook]. I can press a little smiley face. Anything where I can just press a button, but I am no good at this typing thing at all ... mostly and my hands shaking, they tend to want to press the wrong button and I have to start all over again. (P002, female, 78 years)

To be honest my hands aren't steady enough to press the little button to send messages. (P018, female, 78 years)

There is an important consideration for the interface of digital devices and social media as it can have implications for use.

\section{Technology as a tool for social engagement}

Technology as a connector

Many individuals discussed the benefits of digital devices, social technology and social applications as a method of connecting with others. Primarily, these 
technologies were used as a way to connect with family and friends living geographically dispersed, particularly those living abroad:

I mean a lot of them are from a long, long, way, away and you're not likely to see them. I mean there is one in [another country]. So yes, it's nice just to get some news. (P010, female, 74 years)

In addition to the use of social technology to keep in touch with those living geographically dispersed, increased frequency of communication was also a benefit of online communication, with participants describing how they would 'throw things at each other' (P017, male, 76 years) using online social technology as opposed to less-consistent offline communication:

[My children and I don't] communicate too often, but with a WhatsApp family page and things we throw things at each other. We've been hearing about my daughter's holiday. My son is abroad at the moment, we've been hearing about him. He works abroad quite a lot, so he keeps in touch that way. (P017, male, 76 years)

Social technology was predominantly used to connect with their existing network of family and friends, and they discussed the importance of 'meaningful' relationships online, rather than conversing with people they did not know, or without reason:

Unless there is a possibility of some connection, some point of it then I don't care. I don't need thousands of so-called friends. (P003, male, 70 years)

P003 described the importance of using social technology to develop real connection, rather than simply being connected with others. P003 used social media as a means of enhancing existing relationships, akin to the stimulation hypothesis (Gross, 2004; Valkenburg and Peter, 2007). This use of social technology for connection was also experienced by another participant, a carer, who described himself as 'almost housebound' (P012, male, 71 years). This participant found social media to be a useful way to connect with others, however, he very much relied on this form of social connection as without it he would no longer get to socialise with others. This not only allows him to socialise with others whilst being at home, but also allows him to socialise in a way that he enjoys:

Social isolation is one of the big problems and to know that there is someone that you can just greet and say something to when you get up in the morning is helpful. (P012, male, 71 years)

P012 has begun meaningful relationships using Twitter and describes the caring environment that can be created on the social media platform. However, one issue with this form of communication is that these discussions are inconsistent and unreliable: 
Well it's just on Twitter so if they come up, they come up. They're all in the same place, so if they say something I might respond. If they're not there then if I don't hear anything for a couple of days I might say, 'Is everything okay?' (P012, male, 71 years)

In many cases, P012 displaced his offline relationships with online relationships through circumstance and caring responsibilities. However, unlike face-to-face communication, social internet use is inconsistent and unreliable, having the potential to increase experiences of loneliness.

Similarly, others did not always perceive the use of digital devices, social technology or the social internet as being a choice. Participants often felt that their own choice was removed, as using social media sites is now necessary to remain connected with others:

But [I use Facebook] just to keep a check and see exactly what they are all up to you know, otherwise I just don't know what is happening and I do feel a bit cut off. (P002, female, 78 years)

Individuals also expressed concern for those without digital skills or access to digital devices. One participant described this as 'those that can and those that can't' (P003, male, 70 years). The benefits experienced due to their own use of technology was recognised, and participants were concerned for others without such access:

A lot of people seem to communicate a lot via technology, rather than actually communicating directly and meeting up ... and that can be quite isolating, especially for older people, I think ... a lot of people I know that are older than me, around my age and older than me, they don't have computers ... so yeah, I think a lot of older people become more isolated because as other younger people tend to use a lot of computers and a lot of phone messages, and I think older people can become quite isolated. (P011, female, 74 years)

Generally, social technologies, more specifically, social media, text messaging and video calling, were seen as connectors, and those who did not use this technology could be left out. However, despite the perceived benefits of social technology, some individuals did acknowledge how this in itself could also be isolating:

[The iPad] has it's uses but you do have to watch yourself otherwise you could sit all day on social media and never get out of the house. (P007, female, 72 years)

P007 acknowledges the balance between using digital devices and social technology as an enhancer of social connection and relationships, and displacing offline relationships with online relationships. They describe the pull of social media and potential overuse.

On the whole, participants described the benefits of online communication in allowing them to connect with their family and friends, and both maintain and enhance these offline relationships. 
The importance of the visual

Many participants described online communication as a way to keep in touch with one another when face-to-face communication was not possible. Visual communication using online visual communication tools, such as video calling applications or sharing photographs, was felt to be the mode of online communication offering an opportunity most similar to being there in person. In seeing one another, individuals spoke about 'really' getting a sense of how someone was feeling and connecting with them on a level that was deeper than telephone or other online communication:

I mean it's the visual. If you're having a conversation with someone and you can see their face and see the response and the smiles and the rest, it just adds something, doesn't it? ... And the same with the telephone you can find out that you have an argument starting that wouldn't be there if you could see the twinkle in the eye. (P012, male, 71 years)

Online visual communication tools reduced the reliance on verbal communication, and allowed participants to see body language and facial expressions. Online visual communication was not only important for themselves, but also their family, with one participant describing it as a way for grandchildren not to forget about their grandparents:

My son says, he wants them to keep contact with us [using online visual communication tools] as well, so they don't forget who their grandparents are. (P005, female, 79 years)

However, one participant described the negative consequences of video calling for her young granddaughter:

My daughter has problems with the youngest one, she gets very upset because she can see you but she can't touch you. So, we tend not to do FaceTime a lot, but it is there if you need it. (P004, female, 73 years)

The use of online visual communication tools was not always beneficial, in this case for younger family members. However, another downside to video calling was concern over appearance and body image:

FaceTime. I'm not very keen on it because I have too many wrinkles ... I am not keen on FaceTime, no, unless I am all made up. (P001, female, 83 years)

Unlike other forms of online communication, individuals worried about their appearance, and were self-conscious of how they looked on screen.

In addition to video calling, visual communication in the form of sharing photographs was also of great importance:

It's a wonderful thing, it's a wonderful thing to move photographs around, to chat to people around the world. (P003, male, 70 years) 
Photograph sharing was often passive, and participants mostly described how others sent photographs sent to them, rather than sharing photographs of themselves with others:

I like seeing the others' photographs, as I say I haven't actually done it, sent any myself. (P013, female, 79 years)

There was a passivity in this form of online communication. When an individual did speak of sending photographs to others or showing people photographs stored on their digital devices, this was most often the photographs of their relatives that had been sent to them, rather than photographs of themselves.

Online visual communication was valued by participants as being most like face-to-face communication, although there were some drawbacks to using video calling applications and sharing photographs.

\section{Discussion}

This study aimed to explore older adults' experiences of using technology (including digital devices, social media and social applications) to connect with others. This study involved older adults with existing access to digital devices for online social connection (first-level digital divide), and specifically aimed to understand skills and usage (second-level digital divide), motivators and tangible outcomes (third-level digital divide) of using digital devices and social media as tools to connect with others. The most salient findings from this study illustrated that, even for users with access to and regular use of social technology, a lack of skills or perceived lack of skills acted as barriers to using social technology as a way of communicating with others online. Furthermore, perceptions of social technology for online communication acted as both facilitators and barriers to its use, ultimately influencing the frequency and methods of online communication.

These barriers comprised physical, psychological and social factors. The culture of online communication is a barrier highlighted in this study. Specifically, this has an impact on perceptions of social technology use, and the frequency and method of online communication. All other barriers are reminiscent of general barriers to technology use, namely physical functioning, perceived self-efficacy, fear and social capital. These barriers not only reduce overall use of technology and exacerbate digital exclusion, but also limit the opportunity to use technology for social connection. Furthermore, the general barriers generated in this study emphasise nuances specific to social technology use, e.g. access and use of social media sites, and the influence of body image when using video calling applications.

Much evidence exists to highlight the importance of self-efficacy, fear and social capital within factors of digital exclusion of general technology use (Age UK, 2015, 2018; Tsai et al., 2015; Van Deursen and Helsper, 2015; Friemel, 2016; Centre for Ageing Better, 2018), and barriers related to physical functioning have also been highlighted in the evidence base (Wright and Hill, 2009; Neves et al., 2013; Olphert and Damodaran, 2013; Gell et al., 2015). All of these barriers were pertinent to the experiences of participants in this study. Whilst these barriers were not specific to the use of social technology, this study has added to the evidence base, 
highlighting the additional impact of these barriers in reducing the frequency and quality of online social connection.

One of the barriers most pertinent to older adults' use of social technology was the perceived culture of this online communication. The older technology users involved in this study perceived generational differences in the use of social technology, active and passive styles of communication, as well as moral attitudes to online communication that would not necessarily occur 'offline'. Individuals socially construct meaning to technology over time (Dutton and Reisdorf, 2019). Online 'novices' may sense there is a lack of code of social conduct in online interactions (Leist, 2013) and therefore feel apprehensive about the culture of online communications. Social norms differ online and users frequently exhibit different and sometimes unpleasant behaviours online (Leist, 2013), and therefore internet culture, specifically the culture of social media, can be off-putting. Bakardjieva (2005: 186) proposed a number of typologies of socially connected internet users, and two conceptual dichotomies which shaped the theoretical debate concerning social life on the internet: 'virtual versus real and public versus private'. Whilst this theoretical underpinning of digital social connection was developed before the conception of popular social technology, social media and other social applications, such as messaging applications and video-calling technology, these dichotomies were observed in this current study. Some used social media sites, as well as messaging applications, as a transactional source of information in which they gained social information whilst being reluctant to participate themselves. Even chatting in this instance was simply transactional, and without the want for 'virtual togetherness' (Bakardjieva, 2005); whereas others were seeking sociability, anonymity, social support, recognition and intimacy online, as described by Bakardjieva (2005).

Further participant concerns range from worrying about miscommunication or being taken out of context, to the extreme of revealing too much personal information which can be misused (Xie et al., 2012). Evidence has shown older technology users can perceive the internet as a risky place and this can impact upon technology usage and social media usage (Tsai et al., 2016; Lüders and Brandtzæg, 2017). For example, older adults may feel apprehensive about embracing new technologies or social networks due to negative media attention around data privacy (Xie et al., 2012). Rather than focusing on demographic factors which may exclude individuals from using digital devices and social media, considering internet culture, attitudes and beliefs as factors that can be shaped through intervention is more promising for change (Dutton and Reisdorf, 2019). This also further reinforces the importance of understanding the tangible outcomes that can be derived from internet use.

Within this study, the motivator for technology use was primarily in relation to social connection, and the tangible outcomes primarily centred around social connection, although some experienced more wide-ranging benefits to their technology use. Remaining in contact with family and friends is often perceived as being one of the most important aspects of being online (Age UK, 2015; Tsai et al., 2015; Ferreira et al., 2016). Individuals in this study described the benefits of using social technology as a way to connect with others, especially maintaining meaningful online relationships with people they already knew (Coelho et al., 2017). As a result, social technology was perceived as having various benefits to facilitating existing 
social relationships, such as the ability to connect with others living geographically dispersed as well as an increased frequency of communication, with individuals specifically stating they would feel 'cut off' without the use of technology as a source of communication. However, part of the proposed culture of online communication was illustrated through perceptions of social technology, as despite social connection being a major tangible benefit to using social technology, it was not seen as being a replacement for offline communication, but simply as a supplementary tool. Most participants in this study utilised online tools as supplementary tools to social engagement, rather than being a replacement for face-to-face connection. Furthermore, rather than displacing offline relationships with online relationships, instead they used these tools as a way of bridging face-to-face communication, as illustrated in other studies (Lüders and Brandtzæg, 2017).

It is evident that for most, social technology was used predominantly as a way to enhance existing connections, as opposed to escaping the social world and withdrawing (Gross, 2004; Valkenburg and Peter, 2007; Nowland et al., 2018), which ultimately has the potential to increase satisfaction with social networks and reduce loneliness. Only one participant experienced displacement of offline relationships with online ones, although others acknowledged that this withdrawal from offline activities and social relationships would not be difficult. Nowland et al. (2018) describe that both the stimulation and displacement hypotheses are not mutually exclusive, and this was evident in this study with such discussions. This particular participant experienced isolation through caring responsibilities, which is widely evidenced as being related to loneliness (Carers UK, 2015). Although mostly perceived as positive, there was a displacement of offline with online relationships, and online social interactions were inconsistent and did not replicate face-to-face communication (Kraut et al., 1998; Nie, 2001; Nie et al., 2002; Valkenburg and Peter, 2007).

The use of digital technology as a tool to enhance offline social relationships was further evidenced through the importance placed on online visual communication, as this was most reminiscent of face-to-face communication. However, there were key cultural aspects of visual online communication. Previous evidence suggests the importance of online telecommunication applications, such as FaceTime or Skype (Tsai et al., 2015), and photo sharing as a preferred option for older adults to text (Morris et al., 2005; Tsai et al., 2015) as it can provide a background for conversations with relatives living geographically dispersed (Romero et al., 2007; Harley et al., 2009; Coelho et al., 2017). It was clear that the use of technology, specifically online visual communication tools, for social connection was valuable as an alternative method of social connection, and whilst they still preferred face-to-face communication, technology allowed them to connect with friends and family when this was not possible. However, one barrier that was specific to the use of social technology was the importance of body image which impacted use of visual communication tools as some individuals were conscious of their image on screen. Whilst much research has explored the impact of social media on body image, to date there is no research evidencing this as part of video calling platforms and its impact on use.

The proposed physical, psychological and social barriers emphasise the multifaceted barriers experienced by older adults' using social technology. However, is not intended to accentuate the individual's own role and responsibilities in 
adapting to technology and digital services. Rather, it intends to highlight the multi-faceted barriers that older technology users continue to face, and barriers that should be considered when designing technology for social connection. A recently published report written by the Science and Technology Committee (2021) recognises a significant barrier to the uptake of technologies is that older adults are not involved in product design, and therefore the report recommends that the input of older adults in technology design is essential to ensure that this technology address the right issues. One solution to address this is through inclusively designing and/or co-designing digital devices, social media sites and social applications with older people. This will help to improve design and usability, which will in turn maximise regular use, minimise the potential to disengage and promote the use of technology for social connection.

Finally, whilst this study was carried out before the onset of the COVID-19 pandemic, the findings from this study are pertinent to the crisis. Since the start of the COVID-19 pandemic, many have relied on technology more than usual as individuals are unable to communicate with others face to-face. Using these technologies has allowed individuals to maintain connections with others outside their home. But not everyone has access to these tools to support their daily living and wellbeing at home, and as described throughout this paper, as part of the discussion around physical, psychological and social facilitators and barriers of older adults' use of social technology, even those with access to this technology may not have access to the necessary platforms or the skills to use these platforms. There is a divide between those able to access and those excluded from online support. The reliance on technology during the COVID-19 pandemic has brought the 'digital divide' to the fore. Reliance upon one's social capital is limited due to not being able to see others, and it is important to consider those who are no longer able to rely on social networks like friends or family for help in using technology. This lack of access significantly heightens inequalities for so many people in all different ways, and a more digitally centred world is not necessarily achievable for everyone. It is vital that those who struggle to use technology or get online for reasons of social connection and beyond are not left behind.

\section{Strengths and limitations}

While the study is relatively small, a strength of this paper is that participants were recruited from across England, Scotland and Wales, meaning we were able to draw upon experiences from a number of geographical regions. Education level, socioeconomic status and health status were not collected as demographic information, and neither were measures of loneliness or social isolation. This is a limitation as they are all evidenced factors impacting on digital exclusion and would have given more insight into the sample included in the study. One further limitation of this research is the absence of ethnic diversity in the participant sample. Future research should take a purposive sampling approach in order to address this limitation. Additionally, future research should consider including participants who are entirely 'digitally excluded' in order to gain the perspectives of those who do not have any online presence. 


\section{Conclusion}

Digital exclusion exists as three distinct levels: access, skills and usage, and tangible offline benefits derived from technology use. It is clear from this study that despite access to technology for online communication, digital exclusion to social technology can still be experienced through physical, psychological and social barriers, and perceptions of social technology and online communication. Many of these barriers replicate barriers to more general technology use, with some nuances; however, culture around online communication and the influence of body image were novel and specific to social technology use. When new technologies and services are being developed, or digital connection is being promoted, it is important that individuals with digital access are considered as being part of those experiencing digitally exclusion and are eligible for support. These barriers to skills and usage reduce the use of technology, significantly heightening inequalities in various ways, even those with access to this technology.

Individuals saw merit in using technology and social media to connect with others, and social connection was most often considered a valuable tangible offline benefit to technology use. Yet individuals also recognised the detriment of online social connection, particularly when it was considered the only option. If devising interventions or support services for social connection, it is important to incorporate digital communication as one part of a wide-ranging programme. Technology is not a replacement for face-to-face communication but is a supplementary tool when face-to-face communication is not available.

Supplementary material. A podcast has been developed from this study which can be found on: Spotify: https://open.spotify.com/show/3YaugSTw5wTNCujhTZSTug; Soundcloud: https://soundcloud.com/user589740502/ageing-in-a-digital-world; iTunes: https:/podcasts.apple.com/gb/podcast/ageing-in-a-digitalworld/id1506615062?i=1000470663608

Acknowledgements. We would like to thank the British Academy/Leverhulme for the funding which allowed the study to be carried out. We would also like to thank all of the participants who took the time to tell us their stories and experiences. We would like to thank and acknowledge the expertise provided by Amrita Singh, the technical sound expert who produced the podcast accompanying this paper.

Author contributions. GW is principal investigator of this study, conceived the project, assisted in data collection/data analysis and wrote/edited this paper. JRG assisted in data collection/data analysis and co-wrote/edited this paper. SV assisted in data analysis and co-wrote/edited this paper. DJM assisted in data analysis and co-wrote/edited this paper.

Financial support. This work was supported by the British Academy/Leverhulme small research grants (grant number SRG18R1\180818).

Conflict of interest. The authors declare no conflicts of interest.

Ethical standards. Ethical approval was received from Northumbria University's ethical approval system before any data collection was carried out.

\section{References}

Age UK (2015) Later Life in a Digital World. London: Age UK.

Age UK (2018) Digital Inclusion Evidence Review. London: Age UK.

Bakardjieva M (2005) Internet Society: The Internet in Everyday Life. London: Sage.

Ballantyne A, Trenwith L, Zubrinich S and Corlis M (2010) 'I feel less lonely': what older people say about participating in a social networking website. Quality in Ageing and Older Adults 11, 25-35. 
Beneito-Montagut R, Cassián-Yde N and Begueria A (2018) What do we know about the relationship between internet-mediated interaction and social isolation and loneliness in later life? Quality in Ageing and Older Adults 19, 14-30.

Bergström A (2017) Digital equality and the uptake of digital applications among seniors of different age. Nordicom Review 38, 79-91.

Berkman LF, Glass T, Brissette I and Seeman TE (2000) From social integration to health: Durkheim in the new millennium. Social Science \& Medicine 51, 843-857.

Blank G and Groselj D (2014) Dimensions of Internet use: amount, variety, and types. Information, Communication \& Society 17, 417-435.

Braun V and Clarke V (2006) Using thematic analysis in psychology. Qualitative Research in Psychology 3, 77-101.

Büchi M, Just N and Latzer M (2016) Modeling the second-level digital divide: a five-country study of social differences in Internet use. New Media \& Society 18, 2703-2722.

Burholt V, Winter B, Aartsen M, Constantinou C, Dahlberg L, Feliciano V, De Jong Gierveld J, van Regenmortel SV and Waldegrave C (2020) A critical review and development of a conceptual model of exclusion from social relations for older people. European Journal of Ageing 17, 3-19.

Carers UK (2015) Alone and Caring: Isolation, Loneliness and the Impact of Caring on Relationships. London: Carers UK.

Centre for Ageing Better (2018) The Digital Age: New Approaches to Supporting People in Later Life Get Online. London: Centre for Ageing Better.

Choi NG and Dinitto DM (2013) The digital divide among low-income homebound older adults: Internet use patterns, eHealth literacy, and attitudes toward computer/Internet use. Journal of Medical Internet Research 15, e93.

Coelho J, Rito F and Duarte C (2017) 'You, me \& TV' - fighting social isolation of older adults with Facebook, TV and multimodality. International Journal of Human-Computer Studies 98, 38-50.

Cornejo R, Tentori M and Favela J (2013) Enriching in-person encounters through social media: a study on family connectedness for the elderly. International Journal of Human-Computer Studies 71, 889-899.

Cotten SR, Anderson WA and McCullough BM (2013) Impact of internet use on loneliness and contact with others among older adults: cross-sectional analysis. Journal of Medical Internet Research 15, e39.

Cresci MK and Jarosz PA (2010) Bridging the digital divide for urban seniors: community partnership. Geriatric Nursing 31, 455-463.

Creswell JW, Plano Clark VL, Gutmann ML and Hanson WE (2003) Advanced mixed methods research designs. In Tashakkori A and Teddlie C (eds), Handbook of Mixed Methods in Social and Behavioral Research. Thousand Oaks, CA: Sage, pp. 209-240.

Dutton WH and Reisdorf BC (2019) Cultural divides and digital inequalities: attitudes shaping Internet and social media divides. Information, Communication \& Society 22, 18-38.

Eurostat (2017) Digital Economy and Society in the EU. Available at https://ec.europa.eu/eurostat/cache/ infographs/ict/images/pdf/pdf-digital-eurostat-2017.pdf.

Ferreira SM, Sayago S and Blat J (2016) Going beyond telecenters to foster the digital inclusion of older people in Brazil: lessons learned from a rapid ethnographical study. Information Technology for Development 22, 26-46.

Friemel TN (2016) The digital divide has grown old: determinants of a digital divide among seniors. New Media e Society 18, 313-331.

Gell NM, Rosenberg DE, Demiris G, LaCroix AZ and Patel KV (2015) Patterns of technology use among older adults with and without disabilities. The Gerontologist 55, 412-421.

Gilleard C and Higgs P (2008) Internet use and the digital divide in the English Longitudinal Study of Ageing. European Journal of Ageing 5, 233-239.

Gordon NP and Hornbrook MC (2018) Older adults' readiness to engage with eHealth patient education and self-care resources: a cross-sectional survey. BMC Health Services Research 18, 220.

Government Digital Service (2014) Policy Paper: Government Digital Inclusion Strategy. London: Cabinet Office.

Gracia E and Herrero J (2009) Internet use and self-rated health among older people: a national survey. Journal of Medical Internet Research 11, e49.

Gross EF (2004) Adolescent Internet use: what we expect, what teens report. Journal of Applied Developmental Psychology 25, 633-649. 
Hargittai E and Dobransky K (2017) Old dogs, new clicks: digital inequality in skills and uses among older adults. Canadian Journal of Communication 42, 195-212.

Hargittai E, Piper AM and Morris MR (2019) From internet access to internet skills: digital inequality among older adults. Universal Access in the Information Society 18, 881-890.

Harley DA, Kurniawan SH, Fitzpatrick G and Vetere F (2009) Age matters: bridging the generation gap through technology-mediated interaction. Extended Abstracts on Human Factors in Computing Systems, pp. 4799-4802.

Heo J, Chun S, Lee S, Lee KH and Kim J (2015) Internet use and well-being in older adults. Cyberpsychology, Behavior, and Social Networking 18, 268-272.

Holttum S (2016) Do computers increase older people's inclusion and wellbeing? Mental Health and Social Inclusion 20, 6-11.

Ihm J and Hsieh YP (2015) The implications of information and communication technology use for the social well-being of older adults. Information, Communication \& Society 18, 1123-1138.

Kraut R, Patterson M, Lundmark V, Kiesler S, Mukophadhyay T and Scherlis W (1998) Internet paradox: a social technology that reduces social involvement and psychological well-being? American Psychologist 53, 1017-1031.

Leist AK (2013) Social media use of older adults: a mini-review. Gerontology 59, 378-384.

Lüders M and Brandtzæg PB (2017) 'My children tell me it's so simple': a mixed-methods approach to understand older non-users' perceptions of Social Networking Sites. New Media \& Society 19, 181-198.

Marino S (2015) Making space, making place: digital togetherness and the redefinition of migrant identities online. Social Media + Society 1. Available at https://doi.org/10.1177/2056305115622479.

Matthews K, Nazroo J and Marshall A (2019) Digital inclusion in later life: cohort changes in internet use over a ten-year period in England. Ageing \& Society 39, 1914-1932.

Morris MG, Venkatesh V and Ackerman PL (2005) Gender and age differences in employee decisions about new technology: an extension to the theory of planned behavior. IEEE Transactions on Engineering Management 52, 69-84.

Neves BB, Amaro F and Fonseca JR (2013) Coming of (old) age in the digital age: ICT usage and nonusage among older adults. Sociological Research Online 18, 1-14.

Nie NH (2001) Sociability, interpersonal relations, and the Internet: reconciling conflicting findings. American Behavioral Scientist 45, 420-435.

Nie NH, Hillygus DS and Erbring L (2002) Internet use, interpersonal relations, and sociability. In Wellman B and Haythornwaite C (eds), The Internet in Everyday Life. Malden, MA: Blackwell, pp. 215-243.

Nowland R, Necka EA and Cacioppo JT (2018) Loneliness and social internet use: pathways to reconnection in a digital world? Perspectives on Psychological Science 13, 70-87.

Office for National Statistics (2018) Internet Users. Available at https://www.ons.gov.uk/businessindustryandtrade/itandinternetindustry/bulletins/internetusers/2018.

Olphert W and Damodaran L (2013) Older people and digital disengagement: a fourth digital divide? Gerontology 59, 564-570.

Pennington CR, Heim D, Levy AR and Larkin DT (2016) Twenty years of stereotype threat research: a review of psychological mediators. PLOS ONE 11, e0146487.

Romero N, Markopoulos P, Van Baren J, De Ruyter B, Ijsselsteijn W and Farshchian B (2007) Connecting the family with awareness systems. Personal and Ubiquitous Computing 11, 299-312.

Scheerder A, van Deursen A and van Dijk J (2017) Determinants of Internet skills, uses and outcomes. A systematic review of the second- and third-level digital divide. Telematics and Informatics 34, 1607-1624.

Schejter AM and Tirosh N (2015) 'Seek the meek, seek the just': social media and social justice. Telecommunications Policy 39, 796-803.

Science and Technology Committee (2021) Ageing: Science, Technology, and Healthy Living. London: House of Lords.

Slegers K, Van Boxtel MP and Jolles J (2008) Effects of computer training and Internet usage on the wellbeing and quality of life of older adults: a randomized, controlled study. Journals of Gerontology: Psychological Sciences and Social Sciences 63B, P176-P184.

Smith A and Anderson M (2018) Social Media Use in 2018. Washington, DC: Pew Research Center. 
Tsai HH, Tsai Y-F, Wang H-H, Chang Y-C and Chu HH (2010) Videoconference program enhances social support, loneliness and depressive status of elderly nursing home residents. Aging and Mental Health 14, 947-954.

Tsai HYS, Shillair R, Cotten SR, Winstead V and Yost E (2015) Getting grandma online: are tablets the answer for increasing digital inclusion for older adults in the U.S.? Educational Gerontology 41, 695-709.

Tsai HYS, Jiang M, Alhabash S, LaRose R, Rifon NJ and Cotten SR (2016) Understanding online safety behaviors: a protection motivation theory perspective. Computers \& Security 59, 138-150.

Tsai JM, Cheng MJ, Tsai HH, Hung SW and Chen YL (2019) Acceptance and resistance of telehealth: the perspective of dual-factor concepts in technology adoption. International Journal of Information Management 49, 34-44.

Valkenburg PM and Peter J (2007) Online communication and adolescent well-being: testing the stimulation versus the displacement hypothesis. Journal of Computer-mediated Communication 12, $1169-1182$.

Van Deursen AJ and Helsper EJ (2015) The third-level digital divide: who benefits most from being online? In Robinson L, Cotten SR and Schulz J (eds), Communication and Information Technologies Annual. Bingley, UK: Emerald Group Publishing, pp. 29-52.

White H, McConnell E, Clipp E, Bynum L, Teague C, Navas L, Craven S and Halbrecht H (1999) Surfing the net in later life: a review of the literature and pilot study of computer use and quality of life. Journal of Applied Gerontology 18, 358-378.

White H, McConnell E, Clipp E, Branch LG, Sloane R, Pieper C and Box TL (2002) A randomized controlled trial of the psychosocial impact of providing internet training and access to older adults. Aging \& Mental Health 6, 213-221.

Winstead V, Anderson WA, Yost EA, Cotten SR, Warr A and Berkowsky RW (2013) You can teach an old dog new tricks: a qualitative analysis of how residents of senior living communities may use the web to overcome spatial and social barriers. Journal of Applied Gerontology 32, 540-560.

Wright DW and Hill TJ (2009) Prescription for trouble: Medicare Part D and patterns of computer and internet access among the elderly. Journal of Aging \& Social Policy 21, 172-186.

Xie B (2007) Information technology education for older adults as a continuing peer-learning process: a Chinese case study. Educational Gerontology 33, 429-450.

Xie B, Watkins I, Golbeck J and Huang M (2012) Understanding and changing older adults' perceptions and learning of social media. Educational Gerontology 38, 282-296.

Yoon H, Jang Y, Vaughan PW and Garcia M (2020) Older adults' Internet use for health information: digital divide by race/ethnicity and socioeconomic status. Journal of Applied Gerontology 39, 105-110.

Yu RP, Ellison NB, McCammon RJ and Langa KM (2016) Mapping the two levels of digital divide: Internet access and social network site adoption among older adults in the USA. Information, Communication \& Society 19, 1445-1464.

Cite this article: Wilson G, Gates JR, Vijaykumar S, Morgan DJ (2023). Understanding older adults' use of social technology and the factors influencing use. Ageing \& Society 43, 222-245. https://doi.org/10.1017/ S0144686X21000490 